\title{
$\log 10$
}

National Cancer Institute

\section{Source}

National Cancer Institute. $\log 10$. NCI Thesaurus. Code C75941.

The base 10 logarithmic value of argument $\mathrm{X}$. 